\title{
KRISEN
}

\section{Vorbeugen ist besser als heilen}

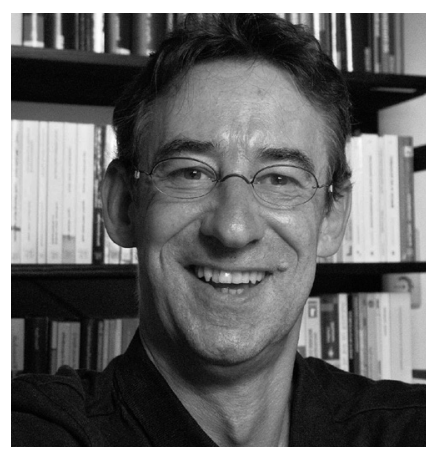

VON HARALD CHRISTA

Prof. Dr. rer. pol. Harald Christa ist Hochschullehrer für Sozialmanagement an der Evangelischen Hochschule Dresden sowie Berater und Gesellschafter der xit $\mathrm{GmbH}$ forschen. planen.beraten. in Nürnberg, Dresden und Berlin.

\author{
In Krisen einer Organisation oder eines Unternehmens \\ lastet auf den Verantwortlichen mitunter ein \\ immenser Druck, der in manchen Fällen kaum noch ein \\ unbefangenes rationales Handeln zulässt. Deshalb gilt \\ es sich immer wieder die Erkenntnis ins Gedächtnis zu \\ rufen, dass es besser ist, eine Krise zu vermeiden, als \\ sie noch so gut zu managen. Ein neues Buch hilft beim \\ Verstehen und beim Vorbereiten auf den Fall des Falles.
}

Eine Krise bedeutet laut Duden eine »schwierige Lage, Situation, Zeit [die den Höhe- und Wendepunkt einer gefährlichen Entwicklung darstellt] «. Krisen sind demnach verbunden mit "Schwierigkeit, kritische Situation; Zeit der Gefährdung, des Gefährdetseins«. (1)

Eine Krise, so heißt es weiter bei Wikipedia, vermittelt bei den Betroffenen ein "Gefühl der Bedrohung «, einen "Anstieg an Unsicherheit", des Weiteren »Dringlichkeit und Zeitdruck und das Gefühl, das Ergebnis sei von prägendem Einfluss auf die Zukunft. Außerdem haben es die Entscheidungsträger oft mit unvollständiger oder verfälschter Information zu tun. Auf emotionaler Ebene entsprechen ihr Verzweiflung oder unkontrollierbarer Zorn ... (2) In aller Munde ist zurzeit die Euro-Krise. Fast täglich können wir die beschriebenen Phänomene über die Medien vermittelt beobachten. Das Vertrauen der Bürgerinnen und Bürger nimmt ab.

Mit dem Stichwort »Krise einer sozialen Organisationen « assoziieren wir im Management in der Regel ebenfalls zunächst einmal eine finanzielle Schieflage eines Verbandes, eines Trägers oder einer Einrichtung. Krise und Geld sind nicht notwendigerweise Zwillinge, aber eng verwandt, denn eine Insolvenz gefährdet das Leben eines Unternehmens. Doch erinnern wir uns an die Schwierigkeiten, in welche UNICEF geraten ist, als ein aufsehenerregender Artikel in der "Frankfurter Rundschau « über vermeintlich wenig stimmige Verwendungspraktiken bei Spendengeldern berichtet hat (3), oder an die Krise der Treberhilfe in Berlin, als staatsanwaltliche Ermittlungen gegen den langjährigen Geschäftsführer geführt wurden und die Öffentlichkeit über dessen Maserati im Wert von 112.060 Euro sowie eine »Jubiläumssonderzahlung « von 80.000 Euro irritiert wurde. (4) Beide Organisationen standen zum Zeitpunkt der Krise finanziell gut da, wurden aber von den Medien wegen vermeintlich anderer Fehlentwicklungen gegeißelt.

Beide Krisen zeichnen sich dadurch aus, dass aus einem vermeintlichen Sommerregen ein die Öffentlichkeit beschäftigender Taifun wurde. Die Krise wurde ganz offensichtlich von den Verantwortlichen der jeweiligen Organisation weder im Entstehen richtig eingeschätzt, noch schnell und hinreichend gedeckt.

Die eigene Erfahrungen des Rezensenten in Beratungsprojekten bestätigen, dass auf den Entscheidungsträgern und Entscheidungsträgerinnen in schweren Phasen einer Krise ein mitunter immenser Druck lastet und in manchen Fällen die Rationalität von Entschlüssen proportional zur Gefahr abnimmt. Heterogene Einschätzungen zur Lage und eine unstrukturierte Kommunikationskultur im Hause erschweren meist die Situation 
noch zusätzlich. Nicht selten fühlte man sich an die Apostelgeschichte 19,32 erinnert: »Dort schrien die einen dies, die anderen das; denn in der Versammlung herrschte großes Durcheinander, und die meisten wussten gar nicht, weshalb man überhaupt zusammengekommen
Organisation, sie schärfen gleichsam den Blick auf das eigene Haus.

Wie bereits angedeutet, ist Krisenmanagement zunächst eine organisatorische Frage. Aus diesem Grunde werden von Adrian Teetz des Weiteren neben typischen Risiken und Fehlerquellen vor

"Führungskräfte sollten sich schon vor einer Krise auch Gedanken über

\section{persönliche Konsequenzen machen"}

war." Zumindest im Fall der Berliner Treberhilfe hatte die Krise nicht nur imageschädigenden Charakter, sondern führte zu einer realen Bestandsbedrohung. So manche Verantwortliche anderer Träger des sozialen Sektors dürften sich zum damaligen Zeitpunkt und vielleicht heute noch gefragt haben, wie sicher sie eigentlich vor einer Krise ihrer Organisation sein können.

Für Vorstandsmitglieder und Geschäftsführende sozialer Verbände, Träger und Einrichtungen könnte mithin eine Publikation von Adrian Teetz zum bestmöglichen Umgang mit Krisen von Organisationen interessant sein. Der Autor konzentriert sich vor allem auf öffentlichkeitswirksame Krisen. Er legt seinen Schwerpunkt auf solche Situationen, in welchen der Verdacht von Fehlverhalten wie Untreue, fachliche Mängel und Ähnliches publik werden und eine Organisation in die Enge getrieben wird. Dabei geht er davon aus, dass insbesondere »diffuse Lagen « für die betroffene Organisation besonders heikel sind. Wie man relevante Fehlentwicklungen erkennen und im Zweifel dagegen steuern kann, damit eine Krise deeskaliert oder am besten gar nicht $\mathrm{zu}$ einer solchen wird, dies sind die hauptsächlichen Fragen, die der Autor in seiner Publikation angeht.

Die inhaltlichen Schwerpunkte konzentrieren sich zunächst auf die Frage, was eine Krise eigentlich ausmacht. Anhand von Fallbeispielen wie Hygienemängel im Krankenhaus werden typische Indikatoren einer Krise sowie der ideale Prozess eines Krisenmanagements aufgezeigt. Natürlich können die Hinweise des Autors keine hundertprozentige Sicherheit geben, aber sie sensibilisieren für krisenhafte Tendenzen in einer (sozialen) allem die strukturellen Voraussetzungen einer erfolgreichen Krisenbewältigung in einer Organisation aufgezeigt. Da sich der bedrohte Ruf einer Organisation gerade im sozialen Bereich verheerend auf das Spendenaufkommen, die Mitgliederzahl, die Motivation der Mitarbeitenden, nicht zuletzt auch auf die Nachfrage von öffentlichen Trägern auswirken kann, geben auch die weiteren Ausführungen und Hinweise dieses Buchs zu Fragen der inhaltlichen Positionierung sowie den Instrumenten einer öffentlichen Stellungnahme im Krisenfalle für die Verantwortlichen sozialer Organisationen wertvolle Hinweise für ein Handling in turbulenten Zeiten.

Auch die Auflistung des Autors zu besonderen Risiken beziehungsweise typischen Fehlerquellen beim Umgang mit Krisen können zur Professionalität einer sozialen Organisation im Handling von Krisen durchaus beitragen. Denn das Spektrum der Fehler und Fettnäpfchen ist weit und reicht von einer Vielstimmigkeit über eine Medienschelte bis hin zur Vermittlung von lebensfremden Standpunkten und Schuldzuweisungskaskaden. Da sich ein organisationaler Notfall auch über einzelne Personen ausrücken kann, sollte der Abschnitt über persönliche Konsequenzen von Entscheidungsträgern ebenfalls nicht überblättert werden.

Wir empfehlen die Lektüre dieses Buches besonders für Einsteigerinnen und Einsteiger, verweisen allerdings explizit darauf, dass mit dem Abschnitt zu Notwendigkeiten und Möglichkeiten der Prävention eines der wichtigsten Kapitel gegen Ende des Werks vorzufinden ist. Wie wir wissen, ist die Verhütung jeder Regulierung eines Schadensfalls vorzuziehen. Insoweit sollte in krisen- freien Zeiten die aktive Arbeit an der Krisenvorbeugung durch klare Regelwerke, eine krisensichere Führungskultur sowie eine gezielte Vorbereitung im oberen und mittleren Management auf problematische Situationen nicht $\mathrm{zu}$ kurz kommen.

Adrian Teetz ist es geglückt, ein leicht lesbares, gleichwohl aber informatives Buch über den richtigen Umgang mit Krisen zu verfassen. Er schreibt erfreulicherweise nicht vom Katheder herunter: Seine Erfahrung in Krisenstäben zeigt sich an vielen Stellen. Das Werk ist gut strukturiert und enthält lehrreiche Praxisbeispiele, auch aus dem Bereich der personenbezogenen sozialen Dienstleistungen. Es ist dem Autor gelungen, so abstrakt wir nötig und so konkret wie möglich den guten Umgang mit Krisen aufzuzeigen. Inhaltlich bereits vorinformierten Managerinnen und Managern können wir nach wie vor die Standardwerke von Hutzschenreuter/Griess-Nega (2006) und den von Feldbauer-Durstmüller/Schlager (2006) herausgegebenen Band empfehlen. Näher mit Fragen der Kommunikationspolitik in der Krise befasst sich das Buch von Joachim Garth (2006).

\section{Anmerkungen}

(1) www.duden.de/rechtschreibung/ Krise\#Bedeutung

(2) http://de.wikipedia.org/wiki/Krise

(3) Frankfurter Rundschau vom 28. November 2007

(4) w w w.spiegel.de/s piegel/ vorab/a-685973.html

Adrian Teetz: Krisenmanagement. Rational entscheiden, entschlossen handeln, klar kommunizieren. Stuttgart 2012.

Feldbauer-Durstmüller, B./Schlager, J. (Hg.): Krisenmanagement. Wien 2006.

Garth, J.: Krisenmanagement und Kommunikation: Das Wort ist Schwert - die Wahrheit Schild. Wiesbaden 2006.

Hutzschenreuter, Th./Griess-Nega, T.: Krisenmanagement: Grundlagen Strategien - Instrumente. Wiesbaden 2006 . 